\title{
AROGYA Intelligent Health Care Application
}

\author{
By Kannangara A.S., Rathnayaka W.G.P.N., Ranasinghe R.A.K.D. \\ \& Halmillawewa C.A. \\ Sri Lanka Institute of Information Technology
} Abstract- People of today pay less attention to their daily diet due to their busy lifestyles. Therefore, there is a great tendency to contract chronic non-communicable diseases. Furthermore, the lack of nutritious meals and daily exercise causes chronic non-communicable diseases in people with no age difference. In our first part, we developed to predict specialization in cardiology using symptoms. However, when we refer to a doctor, we must at least know what specialist should know based on their symptoms. In addition, there is a problem with the recipe. If the pharmacist has misread the prescription given by the doctor, patients can receive bad medications, leading to terrible side effects and even death due to careless writing by the doctor. As a solution, an application function can be proposed that will be developed in the project and the function should be able to improve the readability and intelligibility of the patient with prescription drugs. Therefore, the patient always knows the prescribed medications through the application to avoid the problem mentioned above. According to the 2016 pharmaceutical magazine, there are cholesterol and diabetic patients suffering mainly from chronic non-communicable diseases in Sri Lanka.

\section{GJCST-H Classification: J.3}

Strictly as per the compliance and regulations of:

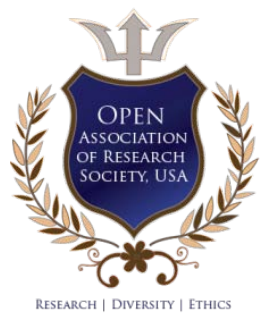

(C) 2021. Kannangara A.S., Rathnayaka W.G.P.N., Ranasinghe R.A.K.D. \& Halmillawewa C.A. This is a research/review paper, distributed under the terms of the Creative Commons Attribution-Noncommercial 3.0 Unported License http://creativecommons.org/licenses/by-nc/3.0/), permitting all non-commercial use, distribution, and reproduction in any medium, provided the original work is properly cited. 


\title{
AROGYA Intelligent Health Care Application
}

\author{
Kannangara A.S. ${ }^{\alpha}$, Rathnayaka W.G.P.N. ${ }^{\circ}$, Ranasinghe R.A.K.D. ${ }^{\rho}$ \& Halmillawewa C.A. ${ }^{\omega}$
}

Abstract- People of today pay less attention to their daily diet due to their busy lifestyles. Therefore, there is a great tendency to contract chronic non-communicable diseases. Furthermore, the lack of nutritious meals and daily exercise causes chronic non-communicable diseases in people with no age difference. In our first part, we developed to predict specialization in cardiology using symptoms. However, when we refer to a doctor, we must at least know what specialist should know based on their symptoms. In addition, there is a problem with the recipe. If the pharmacist has misread the prescription given by the doctor, patients can receive bad medications, leading to terrible side effects and even death due to careless writing by the doctor. As a solution, an application function can be proposed that will be developed in the project and the function should be able to improve the readability and intelligibility of the patient with prescription drugs. Therefore, the patient always knows the prescribed medications through the application to avoid the problem mentioned above. According to the 2016 pharmaceutical magazine, there are cholesterol and diabetic patients suffering mainly from chronic non-communicable diseases in Sri Lanka. This application suggests a healthy and balanced diet for all age differences. By analyzing disease readings, patients will receive their diet, including their interesting meals. A simple blood test called a lipoprotein profile can measure your total cholesterol level. In accordance with our research project, we maintain your medical reports and obtain information about your cholesterol level by the report and give you a prediction on how cholesterol level without drugs, what is the best range and how the level with the drug.

\section{InTRODUCTION}

H uman health is main part of the life. The meaning of health has evolved over time. From the biomedical perspective, early definitions of health focused on the issue of the body's ability to function; health was viewed as a normal functioning state which could be interrupted from time to time by illness. Also, in current era, people too much busy with their works. Because of that, people are suffering with noncommunicable chronic diseases [7]. According to the Pharmaceutical Journal of Sri Lanka 2016 [7], there are Hypertension 48.5\%, Diabetes mellitus $45.3 \%$ and Ischemic Heart disease 29.4\%. Proposed application based on non-communicable chronic diseases. First part mainly based on heart diseases. Because the heart diseases area is wide in the medical world. There are

Author a $\sigma \rho \omega$ : Faculty of computing, Department of information technology, Sri Lanka Institute of information technology, Malabe Sri Lanka.e-mails: anupama.kannangara@gmail.com,

it17040440@my.sliit.lk, it17027120@my.sliit.lk, it16022102@my.slit.lk many categories based on heart diseases named blood vessel diseases, coronary artery disease; heart rhythm problems (arrhythmias); and heart defects born with (congenital heart defects), among others[8]. Also, Cardiovascular disease usually refers to conditions that involve narrowed or blocked blood vessels that can lead to a heart attack, chest pain (angina), or a stroke. Other heart conditions, such as those that affect muscle, valves, or heart rate, are also considered to be forms of heart disease. Other part based on cholesterol, diabetic and blood pressure. Cholesterol is a chemical compound that the body needs as a building block for cell membranes and hormones like estragon and testosterone. The liver produces about $80 \%$ of the body's cholesterol and the rest comes from food sources such as meat, chicken, eggs, fish, and dairy products. Plant-based foods do not contain cholesterol [9]. Cholesterol divided in to three parts known as Highdensity lipoprotein (HDL), Low-density lipoprotein (LDL) and very low-density lipoprotein(VLDL). There are levels known as VLDL $<40 \mathrm{mg} / \mathrm{dL}, \mathrm{LDL}<160 \mathrm{mg} / \mathrm{dL}$, HDL $>=45 \mathrm{mg} / \mathrm{dL}$, Triglycerides $<150 \mathrm{mg} / \mathrm{dL}$.

\section{Method}

\section{a) Data sets}

\section{i. Heart disease prediction model}

The data set that we used in this research, is used various researchers for their research purpose. We get it the web site called kaggle.com[10]. This dataset was used in this research designing for heart disease diagnosis for machine-learning-based system. This heart disease related dataset has a sample size of 4240 patients, 16 features.

\section{ii. Prediction for future cholesterol level model}

The data set that we used to predict cholesterol level is a created data set by me. This data set used in this research for predict cholesterol level for future six months suing time series analysis. This cholesterol level related dataset has a sample size of 20 months of one patient.

\section{iii. Model for diet plan}

I created dataset to similar medicine using SPC guidelines. Because there is no dummy, data set to this part. In that case, I met a doctor and created a sample dataset to create the model.

To predict the diabetic level and cholesterol levels part we got the dummy data using https://www.kaggle.com/ website. 
iv. Give an idea of the prescription

Collected cholesterol prescription as an image data set and created a stranded dataset.

\section{b) Data processing}

There is feature called education. That one is not related to the heart disease. Therefore, we drop that feature. During the cleaning, remove null values. Some null values fill with the mean value of the feature and get a value, which will increase the efficiency. In the prediction of the diabetic and cholesterol level part, started to create the model using jupyter notebook. First, imported necessary libraries and added the dataset. Also dropped the unnecessary data column in the data set.

\section{c) Methodology of the Proposed System}

i. Heart disease prediction model

The proposed system developing with the aim to classify weather people should channel cardiology or not. One of the popular machine learning classifiers logistic regression used for classification of this system. Logistic regression is the one of best classifier to get binary value output. The methodology of the proposed system structured into four stages including (1) preprocessing of dataset, (2) feature selection, (3) machine learning classifiers, and (4) classifiers' performance evaluation methods. Figure 1 shows the framework of the proposed system.

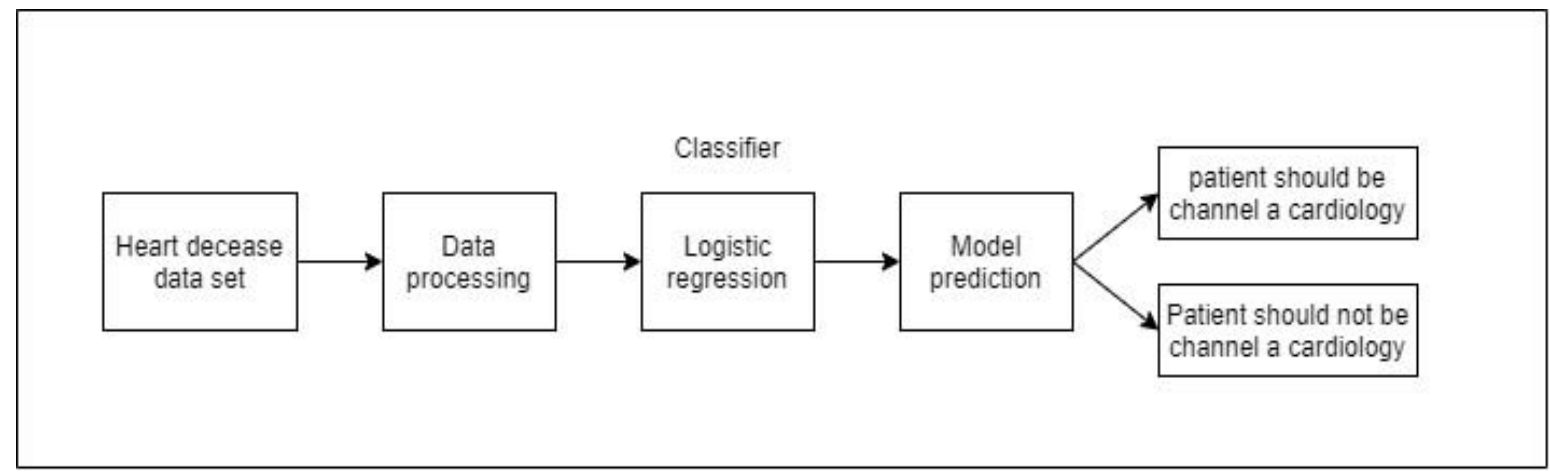

Figure 1

Logistic Regression: A logistic regression is a output is at 0.5 . If the value of hypothesis, it will predict y classification algorithm [27-29]. For binary classification $=1$ which mean that the person has heart disease and problem, in order to predict the value of predictive variable $y$ when $y \in[0,1], 0$ is negative class and 1 is positive class. It also uses multi classification to predict the value of $y$ when $y \in[0,1,2,3]$.

if value of, then predict $\mathrm{y}=0$ which shows that the person is healthy.

Hence, the prediction of logistic regression

In order to classify two classes 0 and $1, a$ hypothesis will be designed and threshold classifier

Logistic regression sigmoid function can be written as follows:

$$
h \theta(x)=g\left(\theta^{T} X\right),
$$

where $g(z)=1 /\left(1+x^{-z}\right)$ and $h \theta(x)=1 /\left(1+x^{-z}\right)$.

Similarly, the logistic regression cost function can be written as follows:

$$
J(\theta)=\frac{1}{m} \sum_{i=1}^{m} \operatorname{cost}\left(h \theta\left(x^{(i)}\right), y^{(i)}\right) .
$$

My ratio is $80-20$. $80 \%$ data will train and $20 \%$ will be test. Import confusion matrix to represent the false positive, false negative, true positive and true negative.

\section{ii. Prediction for future cholesterol level model}

This proposed system developing to predict a cholesterol level for about 6 months of future and store patient past data records of cholesterol level. The time series is one of the popular machine learning prediction algorithms. In time series analysis have one variable at that time. There have an independent variable and a dependent variable. Time series prediction is a form of data mining that predicts future behaviors by analyzing historical data. 
The objectives of a time series prediction ,t is estimated value of $x$ and,$x[t+s]=f(x[t], x[t-1], \ldots ., x[t-N])$, $s>0$ is called the horizon of prediction. Figure 2 shows the prediction of a time series using auto regression integrated moving average (ARIMA-model) [11].

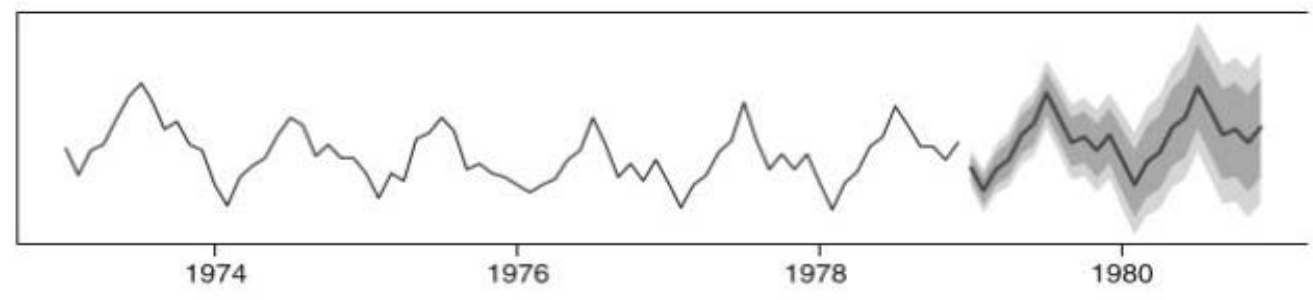

Figure 2

The way a Simply Moving Average is calculated is that it takes the subset of the data mentioned in the moving average model description, adds together the data points, and then takes the average over the subset of data. It can help identify the direction of trends in your data and identify levels of resistance wherein business or trading data. [12]

Forecasting is one of the most relevant tasks when working with time-series data. You can forecast with a simple moving average, another moving average model called 'Autoregressive Integrated Moving Average' is popular for fairly accurate and quick forecasting of time series. The Autoregressive Integrated Moving Average, or ARIMA model, is a linear function that is used for predicting future data points based on past data. ARIMA combines the models the past data points to determine future points to the linear regression model on an independent variable to predict the dependent variable. Because of ARIMA's using past data, a longer series is preferable to get results that are more accurate. [13]

\section{iii. Model for diet plan}

In decision tree classification data model have two main types known as classification tree and regression tree. This is a non-parametric supervised learning method [1]. In this data model, predict the value of the target variable in the data set by learning simple decision rules. In classification, tree outcome was yes/no type. Those decision variables are categorical or discrete. Also, it known as binary recursive partitioning. However, regression tree is taking continuous values or real numbers [2]. There are many different algorithms but in here, mainly used ID3 (Iterative Dichotomies 3) algorithm [3] invented by Ross Quinlan. Simple meaning of this is greedy search via the space of possible branches without no reverse. It is built top-down from a root node and create subsets using similar values. This is known as homogenous [4]. ID3 algorithm used entropy (figure 3) to appraise the homogeneity of the data set.

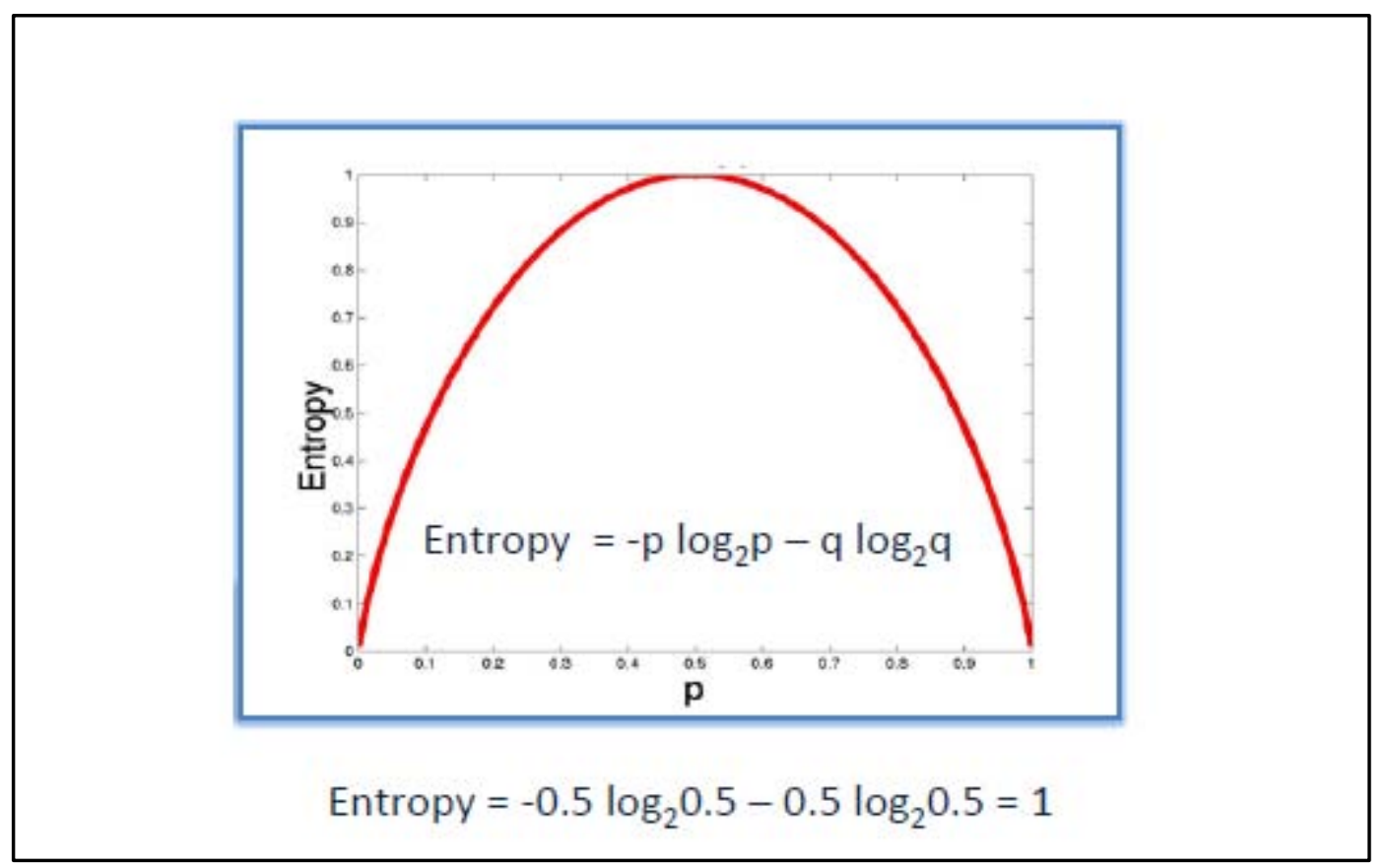

Figure 3 
Entropy using the frequency table to calculate the decision tree within two types. First, one is for one attribute (Figure 4). Second, one is for two attributes
(Figure 5). There are two formulas for above mention types.

$$
E(S)=\sum_{i=1}^{c}-p_{i} \log _{2} p_{i}
$$

Figure 4: Calculation for one attribute

$$
E(T, X)=\sum_{c \in X} P(c) E(c)
$$

Figure 5: Calculation for two attributes

In the similar medicine part, created a histogram to overview the dataset. Finally checked the accuracy of the dataset.

In the cholesterol and diabetic part, create the ranges to diabetic level and cholesterol level. Then created a pie chart to get an idea of the data set. Checked the null values in the dataset. If there were null values, get the mean value of the data column and replaced to the null values. Next, create a histogram to the age column and reading levels. Finally checked the accuracy of the dataset. Next created the meal plan to the reading ranges above mentioned.

iv. Give an idea of the prescription

First recognized of handwritten medical forms.
Algorithm [5]. All lexicon entries are treated as detached words and matched the input word image as containing handwriting to recognize in word model-based recognition. Lexicon entry is the best top choice of this. To develop this model, we created word recognition methodology (Figure 6). Segments are matched against individual characters without using any contextual information in character model-based recognition. In addition, we used Latent Semantic Analysis [6] to compute the relationship between the context of words and terms to a semantic category.

For that, I used Lexicon Driven Word Recognizer

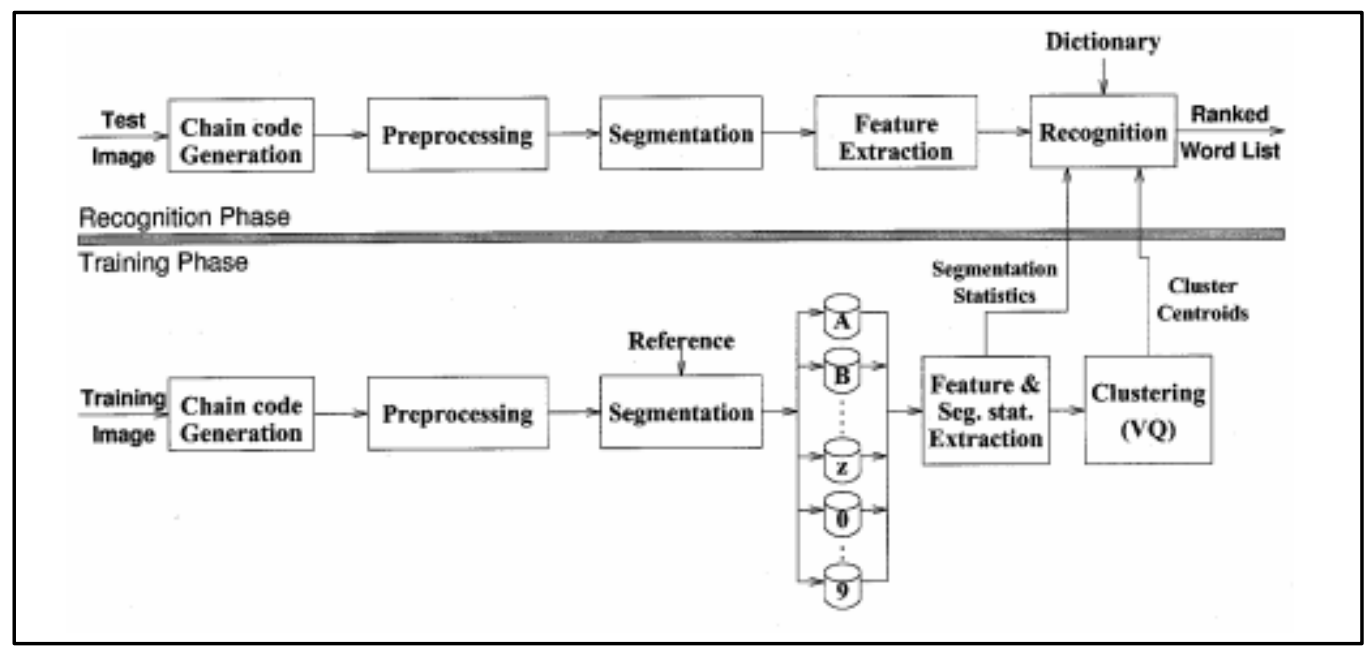

Figure 6

\section{ili. Results and Discussion}

\section{a) Heart disease prediction model}

This section of the paper involved the discussion on the classification model and outcome. First, we checked the performance of machine learning algorithm that I used called logistic regression. All computations were performed on an Intel(R) Core $^{\mathrm{TM}}$ i7 7500U CPU@3.5 GHzPC.

I get the mark $87 \%$ as the result of the accuracy score. Precision score, recall score, $f 1$ score and the confusion metrics would be as follows. 
In $[24]:$

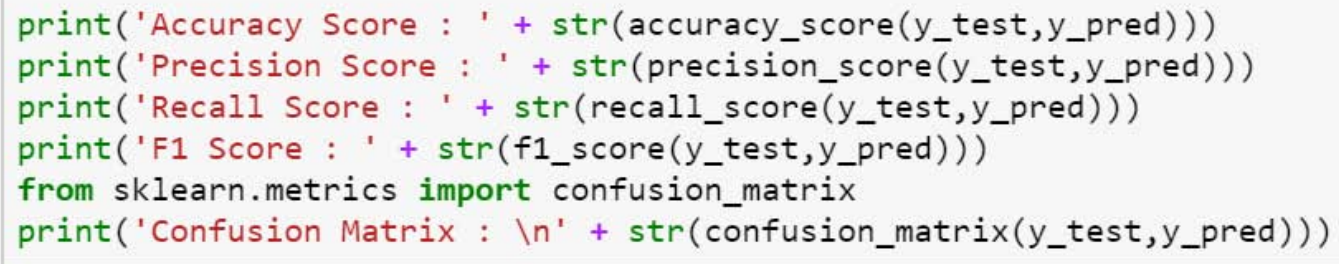

Figure 7

I used grid search for increase my accuracy after the training and testing the data. Accuracy score increased by $0.1 \%$.

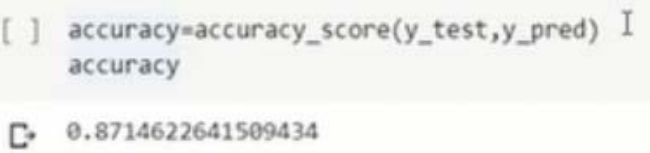

\section{Figure 8}

b) Prediction for future cholesterol level model

For the cholesterol prediction section, we used the performance of time series analysis to predict future cholesterol levels. The result of selecting time series analysis, inputs of cholesterol levels are used to convert to log scale and giving a plot graph and showing prediction line for six months. Other than that in that graph shows the confidence level of prediction

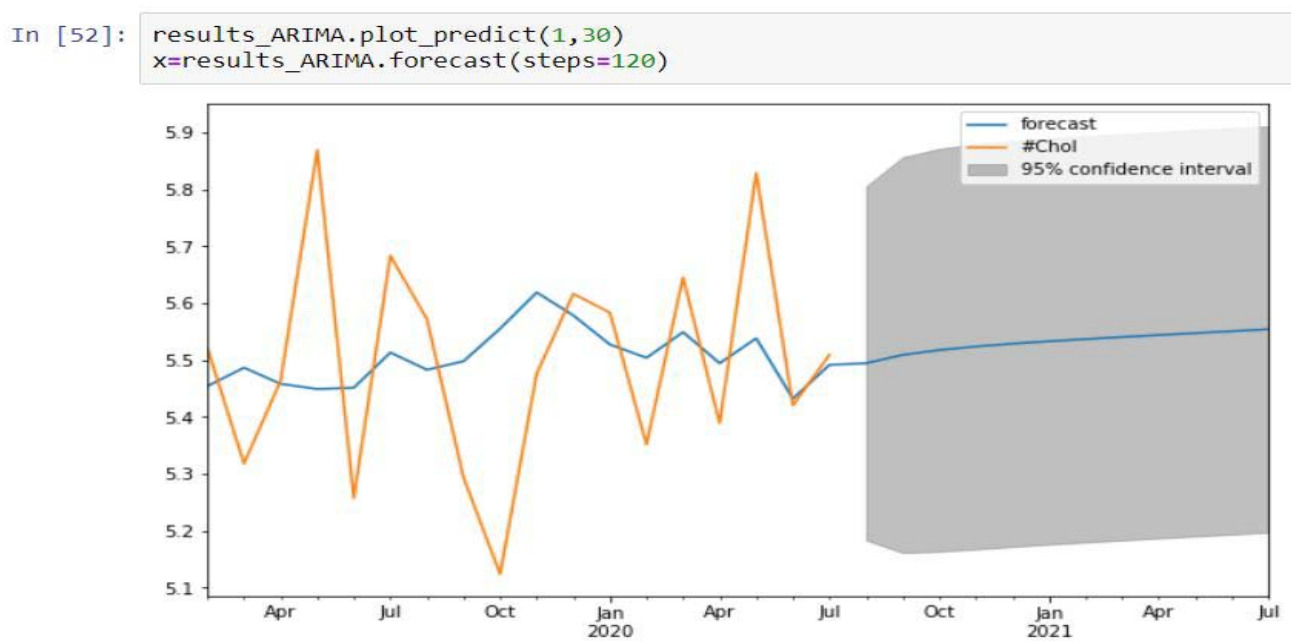

Figure 9

c) Model for diet plan

In the cholesterol level and diabetic level, get the $90 \%$ as accuracy score. Also, predict the result of the data set. As we expected, predict data was the same as the actual data (Figure7). 
In [52]: results_ARIMA.plot_predict (1,30)

$x=$ result's_ARIMA.forecast $($ steps $=120$ )

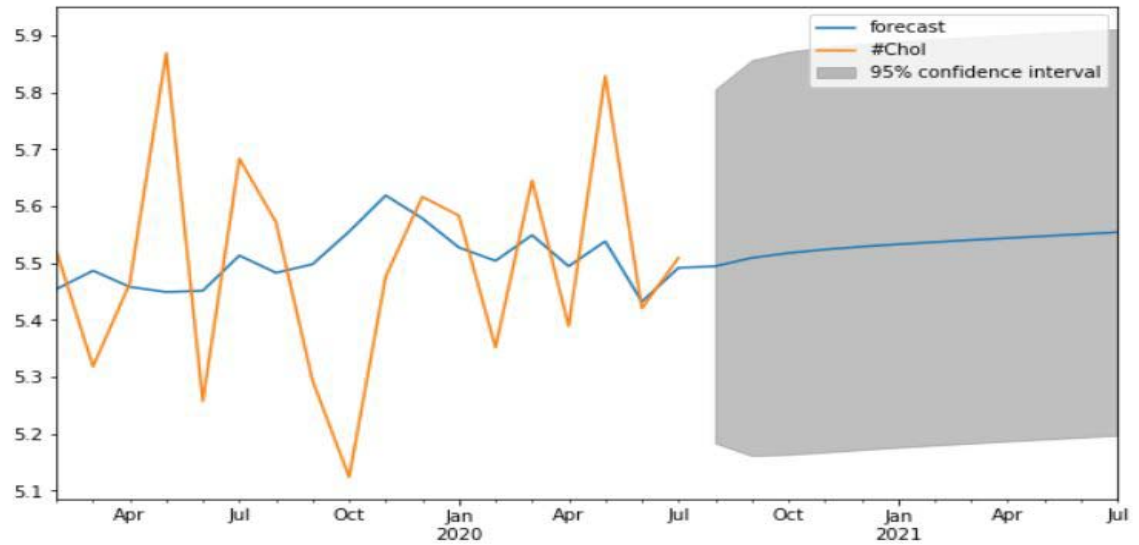

Figure 10

d) Give an idea of the prescription

In the handwriting recognition of the prescription part, get an example to give the result.
Selected a random word as 'word'. Matched the word between a sample image and lexicon entry 'word' (Figure 8).

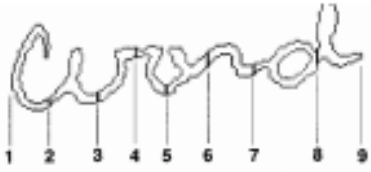

(a) lowest cost path $=5.0+6.1+3.8+4.9=19.8$ confidence of match $=19.8 / 4=4.95$

(b)

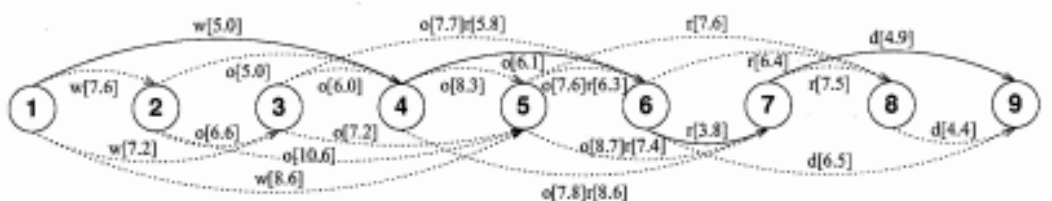

(c)

Figure 11

In the first part, it shows segment point of the image. There are 9 points. Second part is the confidence of the match word. Final part is matching the paths and confidences. For this result will be as the expected one.

\section{Conclusion}

In this research, we try to implement a model for predict heart disease; predict cholesterol and diabetic levels for best meal plan using machine learning algorithm. We train and test model for using given data set. In predict heart disease, part, its accuracy score is $87 \%$ up to now. For that, we used logistic regression for classification. In predict cholesterol and diabetic levels for best meal plan part, its accuracy score is $90 \%$. For that, we used Decision tree for classification.

Researchers can increase accuracy level of the model. However, there are numbers of algorithms to classification. We will perform more experiments to increase the performance of these predictive classifiers for heart disease prediction by using others feature selection algorithms and optimization techniques. If someone follow the heart disease prediction, you all can use different data set and can be use other algorithms for classification. If researchers can implement hybrid model using many algorithms. We think it is also new era of this heart disease prediction model.

In cholesterol level prediction section, we try to implement a model of predict cholesterol level for about six months for future. We train and test model for predict cholesterol level using given dataset and get prediction line and confidence area.

Researchers can develop this model for other diseases and they can try to develop this system using other algorithms and techniques. If someone trying to follow cholesterol level prediction, you can try to get a 
very smooth line using another way for stationarity. If researchers implement this using more algorithm, that also a new thing for cholesterol prediction model.

In prescription reading via image, processing is a big challenge for us. However, using Lexicon Driven Word Recognizer Algorithm, it simplifies the model work. Use of variable duration in word recognition process improved performance.

\section{ACKNOWLedgement}

The Sri Lanka Institute of Information Technology supported for this work.

\section{References Références Referencias}

1. Brownlee, J., 2016. Parametric and Nonparametric Machine Learning Algorithms. [online] Machine Learning Mastery. Available at: https://machinele arningmastery.com/parametric-and-nonparametr icmachine-learningalgorithms/\#: : :text=under lying\% 20mapping\%20function.-,Nonparametric\%20Mach ine\%20Learning\%20Algorithms,form\%20from\%20th e\%20training\%20data.

2. Chakure, A., 2019. Decision Tree Classification. [online] Medium. Available at: https://towardsdata science.com/decision-tree-classification-de64fc4d5 aac

3. En.wikipedia.org. 2020. ID3 Algorithm. [online] Available at: https://en.wikipedia.org/wiki/ID3_ algorithm

4. Saedsayad.com. n.d. Decision Tree. [online] Available at: https://www.saedsayad.com/decision tree.htm\#: : :text=Decision\%20Tree\%20\%2D\%20Cl assification, decision\%20nodes\%20and\%20leaf\%20 nodes

5. Pdfs.semanticscholar.org. 1997. A Lexicon Driven Approach to Handwritten Word Recognition for Real-Time Applications. [online] Available at: https://pdfs.semanticscholar.org/9dde/54b4c73b86 6cde5bdc013ba0de8cf72ee003.pdf

6. Google Books. 2011. Handbook Of Latent Semantic Analysis. [online] Available at: https://books.google. $\mathrm{lk} / \mathrm{books} ? \mathrm{hl}=\mathrm{en} \& \mathrm{l} \mathrm{r}=\& \mathrm{id}=\mathrm{JbzCzPvzpmQC \& oi}=\mathrm{fnd} \&$ $\mathrm{pg}=\mathrm{PP} 1 \& \mathrm{dq}=$ Latent + Semantic + Analysis \&ots $=\mathrm{aN}$ O3G2POHE\&sig =aQIXfzinenum39EpoSmmEwc5R$\mathrm{s} \&$ redir_esc $=\mathrm{y} \# \mathrm{v}=$ onepage $\& \mathrm{q}=$ Latent\%20Semanti $\mathrm{c} \% 20$ Analysis \& $\mathrm{f}=$ false

7. N. Wijekoonand L. Shanika, "Adverse drug reactions and associated factors in a cohort of Sri Lankan patient with non-communicable chronic diseases", ResearchGate.net, 2016. [Online]. Available: https:// www.researchgate.net/publication/307545534_Adv erse_Drug_reactions_and_associated_factors_in_a cohort_of_Sri_Lankan_patients_with_noncommunicable chronic diseases

8. Mayo Clinic staff, "Heart disease-Symptoms and causes", Mayo Clinic, 2018. [Online]. Available: https://www.mayoclinic.org/diseases-conditions/ heart-disease/symptoms-causes/syc-20353118

9. R.Charles Patrick Davis and Benjamin Wedro, "What Is Cholesterol? HDL and LDL Ranges and Diet", Medicine Net, 2016. [Online]. Available: https:// www.medicinenet.com/cholesterol_management/art icle.htm\#why_is_high_cholesterol_dangerous

10. Kaggle.com. 2020. Framingham Heart Study Dataset. [Online] Available at: <https://www. kaggle.com/amanajmera1/framingham-heart-studydataset> [Accessed 17 July 2020].

11. https://www.springer.com/gp/book/9789812874108

12. https://algorithmia.com/blog/introduction-to-timeseries

13. A. M. De Silva and P. H. W. Leong, Grammar-Based Feature Generation for Time-Series Prediction, Springer, Berlin, Germany, 2015.

14. https://machinelearningmastery.com/time-seriesforecasting/

15. https://towardsdatascience.com/the-completeguide-to-time-series-analysis-and-forecasting70d476bfe775 